\title{
An Inertial Iterative Algorithm to Find Common Solution of a Split Generalized Equilibrium and a Variational Inequality Problem in Hilbert Spaces
}

\author{
Mohammad Farid (D), ${ }^{1}$ Rehan Ali, ${ }^{2}$ and Watcharaporn Cholamjiak ${ }^{3}{ }^{3}$ \\ ${ }^{1}$ Department of Mathematics, Deanship of Educational Services, Qassim University, Buraidah 51452, Saudi Arabia \\ ${ }^{2}$ Department of Mathematics, Jamia Millia Islamia, New Delhi 110025, India \\ ${ }^{3}$ School of Science, University of Phayao, Mae Ka 56000, Phayao, Thailand
}

Correspondence should be addressed to Watcharaporn Cholamjiak; watcharaporn.ch@up.ac.th

Received 13 October 2021; Accepted 2 November 2021; Published 29 November 2021

Academic Editor: Jen-Chih Yao

Copyright (c) 2021 Mohammad Farid et al. This is an open access article distributed under the Creative Commons Attribution License, which permits unrestricted use, distribution, and reproduction in any medium, provided the original work is properly cited.

\begin{abstract}
In this paper, we introduce and study an iterative algorithm via inertial and viscosity techniques to find a common solution of a split generalized equilibrium and a variational inequality problem in Hilbert spaces. Further, we prove that the sequence generated by the proposed theorem converges strongly to the common solution of our problem. Furthermore, we list some consequences of our established algorithm. Finally, we construct a numerical example to demonstrate the applicability of the theorem. We emphasize that the result accounted in the manuscript unifies and extends various results in this field of study.
\end{abstract}

\section{Introduction}

Let $H_{1}$ and $H_{2}$ be real Hilbert spaces with inner product $\langle\cdot, \cdot\rangle$ and norm $\|\cdot\|$. Let $C$ and $Q$ be nonempty closed convex subsets of $H_{1}$ and $H_{2}$, respectively. The variational inequality problem (in short, VIP) is to find $x^{*} \in C$ such that

$$
\left\langle B x^{*}, y-x^{*}\right\rangle \geq 0, \quad \forall y \in C,
$$

where $B: C \longrightarrow H_{1}$ is a nonlinear mapping. The solution set of VIP (1) is denoted by $\Omega$. It is introduced by Hartman and Stampacchia [1].

In 1994, Blum and Oettli [2] introduced and studied the following equilibrium problem (in short, EP): find $x^{*} \in C$ such that

$$
G_{1}\left(x^{*}, y\right) \geq 0, \quad \forall y \in C,
$$

where $G_{1}: C \times C \longrightarrow \mathbb{R}$ is a bifunction. The solution set of EP (3) is denoted by Sol (EP(3)).

In the last two decades, EP (2) has been generalized and extensively studied in many directions due to its importance; see, for example [3-7], for the literature on the existence and iterative approximation of solution of the various generalizations of EP (2)

Censor et al. [8] introduced the split feasibility problem (in short, $S_{P} \mathrm{FP}$ ) in finite-dimensional Hilbert spaces for modelling of inverse problems that arise from phase retrievals and in medical image restoration as

$$
\text { find } x^{*} \in C \text { such that } B x^{*} \in Q \text {, }
$$

where $B: H_{1} \longrightarrow H_{2}$ is a bounded linear operator.

In this paper, we consider the following split generalized equilibrium problem (in short, $S_{P} \mathrm{GEP}$ ):

Let $G_{1}, b_{1}: C \times C \longrightarrow \mathbb{R}$ and $G_{2}, b_{2}: Q \times Q \longrightarrow \mathbb{R}$ be nonlinear mappings, and $B: H_{1} \longrightarrow H_{2}$ be a bounded linear operator, then $S_{P}$ GEP is to find $x^{*} \in C$ such that

$$
G_{1}\left(x^{*}, x\right)+b_{1}\left(x, x^{*}\right)-b_{1}\left(x^{*}, x^{*}\right) \geq 0, \quad \forall x \in C,
$$

and such that

$$
\begin{array}{r}
y^{*}=B x^{*} \in Q \text { solves } G_{2}\left(y^{*}, y\right)+b_{2}\left(y, y^{*}\right)-\phi\left(y^{*}, y^{*}\right) \geq 0, \\
\forall y \in Q .
\end{array}
$$


If we take $b_{1}, b_{2} \equiv 0$, then $S_{P}$ GEP becomes split equilibrium problem (in short, $S_{P} \mathrm{EP}$ ) as

$$
G_{1}\left(x^{*}, x\right) \geq 0, \quad \forall x \in C,
$$

and such that

$$
y^{*}=B x^{*} \in Q \text { solves } G_{2}\left(y^{*}, y\right) \geq 0, \quad \forall y \in Q .
$$

When looked separately, (4) is the generalized equilibrium problem (GEP) and we denote its solution set by Sol $(\mathrm{GEP}(4))$. The $S_{P} \mathrm{GEP}(4)$ and (5) constitute a pair of generalized equilibrium problems which have to be solved so that the image $y^{*}=B x^{*}$ under a given bounded linear operator $B$ of the solution $x^{*}$ of the $\operatorname{GEP}(4)$ in $H_{1}$ is the solution of another GEP (4) in another space $H_{2}$. We denote the solution set of GEP (5) by Sol (GEP (5)). The solution set of $S_{P}$ GEP (4) and (5) is denoted by $\Gamma=\{p \in \operatorname{Sol}(\operatorname{GEP}(4)): B p \in \operatorname{Sol}(\operatorname{GEP}(5))\}$.

$S_{P}$ GEP (4) and (5) generalize multiple-sets split feasibility problem. It also includes as special case, the split variational inequality problem, which is the generalization of split zero problems and split feasibility problems, see for details [9-12].

In 2008, Mainge [13] introduced the following inertial Krasnosel'skiľ-Mann algorithm by combining Krasnosel'skil-Mann algorithm and the inertial extrapolation:

$$
\left\{\begin{array}{c}
t_{n}=x_{n}+\theta_{n}\left(x_{n}-x_{n-1}\right), \\
x_{n+1}=\left(1-\eta_{n}\right) t_{n}+\eta_{n} T t_{n},
\end{array}\right\},
$$

for each $n \geq 1$. He proved that the sequence $\left\{x_{n}\right\}$ generated by algorithm (8) converges weakly to a fixed point of $T$ under some conditions. Recently, Bot et al. [14] studied the convergence analysis of the inertial Krasnosel'skiľ-Mann algorithm for approximating a fixed point of nonexpansive mapping $T$ by getting rid of some conditions used in the main result of Mainge [13]. Recently, Dong et al. [15, 16] introduced the inertial hybrid algorithm and established a strong convergence theorem for approximating a fixed point of nonexpansive mapping $T$ in the setting of Hilbert space. For further study of some generalization of iterative algorithm (8), see for instance $[17,18]$. Very recently, Monairah et al. [19] introduced and studied a hybrid iterative algorithm to approximate a common solution of generalized equilibrium problem, variational inequality problem, and fixed point problem in the framework of a 2 uniformly convex and uniformly smooth real Banach space. The inertial method has been studied by many researchers. The results and other related ones analyzed the convergence properties of inertial type algorithms and demonstrated their performance numerically on some imaging and data analysis problems, see for details [20-23].

Motivated by the work given in $[6,13,24]$, we propose an iterative algorithm via inertial and viscosity techniques to find a common solution of a split generalized equilibrium and a variational inequality problem in Hilbert spaces. We obtained the strong convergence for the proposed algorithm. Further, we give some consequences of the main result. Finally, we discuss a numerical example to demonstrate the applicability of the iterative algorithm. The method and result presented in this paper generalize and unify the previously known related methods and results. Our result can extend several iterative methods given in the literature.

\section{Preliminaries}

In this section, we collect some concepts and results which are required for the presentation of the work. Let symbols $\longrightarrow$ and $\rightarrow$ denote strong and weak convergence, respectively.

For every point $x \in H_{1}$, there exists a unique nearest point to $x$ in $C$ denoted by $P_{C} x$ such that

$$
\left\|x-P_{C} x\right\| \leq\|x-y\|, \quad \forall y \in C .
$$

The mapping $P_{C}$ is called the metric projection of $H_{1}$ onto $C$. It is well known that $P_{C}$ is nonexpansive and satisfies

$$
\left\langle x-y, P_{C} x-P_{C} y\right\rangle \geq\left\|P_{C} x-P_{C} y\right\|^{2}, \quad \forall x, y \in H_{1} .
$$

Moreover, $P_{C} x$ is characterized by the fact that $P_{C} x \in C$ and

$$
\left\langle x-P_{C} x, y-P_{C} x\right\rangle \leq 0, \quad \forall y \in C .
$$

This implies that

$\|x-y\|^{2} \geq\left\|x-P_{C} x\right\|^{2}+\left\|y-P_{C} x\right\|^{2}, \quad \forall x \in H_{1}, \forall y \in C$.

In a real Hilbert space $H_{1}$, it is well known that

$$
\begin{array}{r}
\|\lambda x+(1-\lambda) y\|^{2}=\lambda\|x\|^{2}+(1-\lambda)\|y\|^{2}-\lambda(1-\lambda)\|x-y\|^{2}, \\
\forall x, y \in H_{1} \text { and } \lambda \in[0,1],
\end{array}
$$

$$
\|x+y\|^{2} \leq\|x\|^{2}+2\langle y, x+y\rangle, \quad \forall x, y \in H_{1} .
$$

Definition 1 (see [25]). A multivalued mapping $M: H_{1} \longrightarrow$ $2^{H_{1}}$ is called monotone if for all $x, y \in H_{1}, u \in M x$ and $v \in M y$ such that

$$
\langle x-y, u-v\rangle \geq 0 \text {. }
$$

Definition 2 (see [25]). A multivalued monotone mapping $M: H_{1} \longrightarrow 2^{H_{1}}$ is maximal if the graph $(M)$, the graph of $M$, is not properly contained in the graph of any other monotone mapping.

Remark 1. It is known that a multivalued monotone mapping $M$ is maximal if and only if for $(x, u) \in H_{1} \times H_{1}$, $\langle x-y, u-v\rangle \geq 0$, for every $(y, v) \in \operatorname{Graph}(M)$ implies that $u \in M x$.

Lemma 1 (see [26]). Let $\left\{x_{n}\right\}$ and $\left\{u_{n}\right\}$ be bounded sequences in a Banach space $E$ and let $\beta_{n}$ be a sequence in $(0,1)$ with $0<\liminf _{n \rightarrow \infty} \beta_{n} \leq \limsup _{n \rightarrow \infty} \beta_{n}<1$. Suppose $x_{n+1}=(1-$ 
$\left.\beta_{n}\right) y_{n}+\beta_{n} x_{n}$ for all integers $n \geq 0$ and $\limsup _{n \longrightarrow \infty}$ $\left(\left\|y_{n+1}-y_{n}\right\|-\left\|x_{n+1}-x_{n}\right\|\right) \leq 0$. Then,

$$
\lim _{n \longrightarrow \infty}\left\|y_{n}-x_{n}\right\|=0 \text {. }
$$

Lemma 2 (see [27]). Let $\left\{b_{n}\right\}$ be a sequence of nonnegative real numbers such that there exists a subsequence $\left\{b_{n_{i}}\right\}$ of $\left\{b_{n}\right\}$ such that $b_{n_{i}}<b_{n_{i+1}}, \forall i \in \mathbb{N}$. Then, there exists a nondecreasing sequence $\left\{m_{j}\right\}$ of $\mathbb{N}$ such that $\lim _{j \rightarrow \infty} m_{j}=\infty$ and the following properties are satisfied by all (sufficiently large) numbers $j \in \mathbb{N}$

$$
\begin{gathered}
b_{m_{j}} \leq b_{m_{j+1}}, \\
b_{j} \leq b_{m_{j}} .
\end{gathered}
$$

In fact, $m_{j}$ is the largest number $n$ in the set $\{1,2,3, \ldots, j\}$ such that $b_{n}<b_{n+1}$.

Lemma 3 (see [28]). Assume that D is a strongly positive selfadjoint bounded linear operator on a Hilbert space $H_{1}$ with coefficient $\bar{\gamma}>0$ and $0<\rho \leq\|D\|^{-1}$. Then, $\|I-\rho D\| \leq 1-\rho \bar{\gamma}$.

Lemma 4 (see [29]). Assume that $\left\{a_{n}\right\}$ is a sequence of nonnegative real numbers such that

$$
a_{n+1} \leq\left(1-\gamma_{n}\right) a_{n}+\delta_{n}, \quad n \geq 0,
$$

where $\left\{\gamma_{n}\right\}$ is a sequence in $(0,1)$ and $\left\{\delta_{n}\right\}$ is a sequence in $\mathbb{R}$ such that

(i) $\sum_{n=1}^{\infty} \gamma_{n}=\infty$;

(ii) $\limsup _{n \rightarrow \infty}\left(\delta_{n} / \gamma_{n}\right) \leq 0$ or $\sum_{n=1}^{\infty}\left|\delta_{n}\right|<+\infty$.

Then, $\lim _{n \longrightarrow \infty} a_{n}=0$.

Assumption 1. Let $G_{1}: C \times C \longrightarrow \mathbb{R}$ and $b_{1}: C \times C \longrightarrow \mathbb{R}$ be bimappings satisfying the following conditions:

(1) $G_{1}(x, x)=0, \forall x \in C$;

(2) $G_{1}$ is monotone, i.e.,

$$
G_{1}(x, y)+G_{1}(y, x) \leq 0, \quad \forall x, y \in C .
$$

(3) For each $y \in C, x \longrightarrow G_{1}(x, y)$ is weakly upper semicontinuous;

(4) For each $x \in C, y \longrightarrow G_{1}(x, y)$ is convex and lower semicontinuous;

(5) $b_{1}(.,$.$) is weakly continuous and b_{1}(., y)$ is convex;

(6) $b_{1}$ is skew-symmetric, i.e.,

$$
\begin{array}{r}
b_{1}(x, x)-b_{1}(x, y)+b_{1}(y, y)-b_{1}(y, x) \geq 0, \\
\forall x, y \in C .
\end{array}
$$

Now, we define $T_{r}^{\left(G_{1}, b_{1}\right)}: H_{1} \longrightarrow C$ as follows:

$$
\begin{aligned}
T_{r}^{\left(G_{1}, b_{1}\right)}(z)= & \left\{x \in C: G_{1}(x, y)+b_{1}(y, x)-b_{1}(x, x)\right. \\
& \left.+\frac{1}{r}\langle y-x, x-z\rangle \geq 0, \forall y \in C\right\},
\end{aligned}
$$

where $r$ is a positive real number.

Lemma 5 (see [30]). Let $C$ be a nonempty closed convex subset of Hilbert space $H_{1}$. Let $G_{1}, b_{1}: C \times C \longrightarrow \mathbb{R}$ be nonlinear mappings satisfying Assumption 1. Assume that for each $z \in H_{1}$ and for each $x \in C$, there exists a bounded subset $D_{x} \subseteq C$ and $z_{x} \in C$ such that for any $y \in C, D_{x}$,

$$
G_{1}\left(y, z_{x}\right)+b_{1}\left(z_{x}, y\right)-b_{1}(y, y)+\frac{1}{r}\left\langle z_{x}-y, y-z\right\rangle<0 .
$$

Let the mapping $T_{r}^{\left(G_{1}, b_{1}\right)}$ be defined by (21). Then, the following conclusions hold:

(i) $T_{r}^{\left(G_{1}, \phi_{1}\right)}(z)$ is nonempty for each $z \in H_{1}$;

(ii) $T_{r}^{\left(G_{1}, \phi_{1}\right)}$ is single-valued;

(iii) $T_{r}^{\left(G_{1}, \phi_{1}\right)}$ is a firmly nonexpansive mapping, i.e., for all $z_{1}, z_{2} \in H_{1}$,

$$
\begin{aligned}
& \left\|T_{r}^{\left(G_{1}, \phi_{1}\right)}\left(z_{1}\right)-T_{r}^{\left(G_{1}, \phi_{1}\right)}\left(z_{2}\right)\right\|^{2} \\
& \leq\left\langle T_{r}^{\left(G_{1}, \phi_{1}\right)}\left(z_{1}\right)-T_{r}^{\left(G_{1}, \phi_{1}\right)}\left(z_{2}\right), z_{1}-z_{2}\right\rangle .
\end{aligned}
$$

(iv) $\operatorname{Fix}\left(T_{r}^{\left(G_{1}, \phi_{1}\right)}\right)=\operatorname{Sol}(G E P(4))$;

(v) Sol $(G E P(4))$ is closed and convex.

Further, assume that $G_{2}: Q \times Q \longrightarrow \mathbb{R}$ and $b_{2}$ : $Q \times Q \longrightarrow \mathbb{R}$ satisfy Assumption 1. For $s>0$ and for all $u \in H_{2}$, define a mapping $T_{s}^{\left(G_{2}, b_{2}\right)}: H_{2} \longrightarrow Q$ as follows:

$$
\begin{aligned}
T_{s}^{\left(G_{2}, b_{2}\right)}(u)= & \left\{v \in Q: G_{2}(v, w)+b_{2}(w, v)-b_{2}(v, v)\right. \\
& \left.+\frac{1}{s}\langle w-v, v-u\rangle \geq 0, \forall w \in Q\right\} .
\end{aligned}
$$

Then, we easily observe that $T_{s}^{\left(G_{2}, b_{2}\right)}$ is nonempty, singlevalued, firmly nonexpansive, fix $\left(T_{s}^{\left(G_{2}, b_{2}\right)}\right)=\operatorname{Sol}(\operatorname{GEP}(5))$, and Sol (GEP(5)) is closed and convex.

Lemma 6 (see [30]). Let $G_{1}$ and $b_{1}$ satisfy Assumption 1 and let the mapping $T_{r}^{\left(G_{1}, b_{1}\right)}$ be defined by (21). Let $x_{1}, x_{2} \in H_{1}$ and $r_{1}, r_{2}>0$, then

$$
\left\|T_{r_{2}}^{\left(G_{1}, b_{1}\right)}\left(x_{2}\right)-T_{r_{1}}^{\left(G_{1}, b_{1}\right)}\left(x_{1}\right)\right\| \leq\left\|x_{2}-x_{1}\right\|+\frac{\left|r_{2}-r_{1}\right|}{r_{2}}\left\|T_{r_{2}}^{\left(G_{1}, b_{1}\right)}\left(x_{2}\right)-x_{2}\right\| .
$$




\section{Main Result}

In this section, we prove a strong convergence theorem based on the proposed iterative algorithm to approximate a common solution of $S_{P}$ GEP (4), (5), and VIP (1) (Algorithm 1).

Theorem 1. Let $C$ and $Q$ be two nonempty closed convex subsets of Hilbert spaces $H_{1}$ and $H_{2}$, respectively. Let $B: H_{1} \longrightarrow H_{2}$ be a bounded linear operator. Assume that $G_{1}: C \times C \longrightarrow \mathbb{R}, G_{2}: Q \times Q \longrightarrow \mathbb{R}, b_{1}: C \times C \longrightarrow \mathbb{R}$, and $b_{2}: Q \times Q \longrightarrow \mathbb{R}$ are nonlinear mappings satisfying Assumption 1 and $G_{2}$ is upper semicontinuous in the first argument. Assume that $\Theta=\Gamma \cap \Omega \neq \varnothing$. Let $g: H_{1} \longrightarrow H_{1}$ be a contraction mapping with constant $\alpha \in(0,1)$ and $D: C \longrightarrow H_{1}$ be a $\tau$-inverse strongly monotone mapping. Let $\left\{x_{n}\right\}$ be generated by Algorithm 1 and satisfy the following conditions:

(i) $\lim _{n \longrightarrow \infty} \eta_{n}=0, \sum_{n=0}^{\infty} \eta_{n}=\infty$;

(ii) $\lim _{n \longrightarrow \infty}\left(\theta_{n} / \eta_{n}\right)\left\|x_{n}-x_{n-1}\right\|=0$;

(iii) $\left\{\theta_{n}\right\} \subset[0, \theta]$, for some $\theta>0$ and $\delta_{n} \subset(0,2 \tau)$;

(iv) $\liminf _{n \longrightarrow \infty} r_{n}>0$ and $\lim _{n \longrightarrow \infty}\left|r_{n+1}-r_{n}\right|=0$;

(v) $\left\{\lambda_{n}\right\} \subset \mathbb{R}$, such that $a \leq \lambda_{n} \leq b<(1 / L)$, where $L=\|B\|^{2}$.

Then, the sequence $\left\{x_{n}\right\}$ converges strongly to some $q \in \Theta$, where $q=P_{\Theta}(g) q$.

Proof. We divide the proof into several steps.

Step 1. We show that $\left\{x_{n}\right\}$ is bounded. Let $q \in \Theta=\Gamma \cap \Omega$, then $q=T_{r_{n}}^{\left(G_{1}, b_{1}\right)} q \quad$ and $B q=T_{r_{n}}^{\left(G_{2}, b_{2}\right)}(B q)$. Applying the similar steps used in Theorem 1 [6], we obtain

$$
\left\|u_{n}-q\right\|^{2} \leq\left\|t_{n}-q\right\|^{2}-\lambda_{n}\left(1-\lambda_{n} L\right)\left\|\left(T_{r_{n}}^{\left(G_{2}, b_{2}\right)}-I\right) B t_{n}\right\|^{2} .
$$

Thus,

$$
\left\|u_{n}-q\right\| \leq\left\|t_{n}-q\right\|
$$

We estimate

$$
\begin{aligned}
\left\|t_{n}-q\right\| & =\left\|x_{n}-\theta_{n}\left(x_{n-1}-x_{n}\right)-q\right\| \\
& \leq\left\|x_{n}-q\right\|+\theta_{n}\left\|x_{n}-x_{n-1}\right\| \\
& =\left\|x_{n}-q\right\|+\left(\frac{\theta_{n}}{\eta_{n}}\right) \eta_{n}\left\|x_{n}-x_{n-1}\right\| .
\end{aligned}
$$

From condition (ii), $\exists N_{1}>0$ such that

$$
\frac{\theta_{n}}{\eta_{n}}\left\|x_{n}-x_{n-1}\right\| \leq N_{1}, \quad \forall n \geq 1 .
$$

By (27)-(29), we have

$$
\left\|u_{n}-q\right\| \leq\left\|t_{n}-q\right\| \leq\left\|x_{n}-q\right\|+\eta_{n} N_{1} .
$$

Since the mapping $I-\delta_{n} B$ is nonexpansive, therefore

$$
\begin{aligned}
\left\|v_{n}-q\right\| & =\left\|P_{C}\left(I-\delta_{n} D\right) u_{n}-q\right\| \\
& \leq\left\|\left(I-\lambda_{n} D\right) u_{n}-\left(I-\delta_{n} D\right) p\right\| \leq\left\|u_{n}-q\right\| .
\end{aligned}
$$

We estimate

$$
\begin{aligned}
\left\|x_{n+1}-q\right\| & =\left\|\eta_{n} g\left(x_{n}\right)+\left(1-\eta_{n}\right) v_{n}-q\right\| \\
& =\left\|\eta_{n}\left(g\left(x_{n}\right)-q\right)+\left(1-\eta_{n}\right)\left(v_{n}-q\right)\right\| \\
& \leq \eta_{n}\left\|g\left(x_{n}\right)-q\right\|+\left(1-\eta_{n}\right)\left\|v_{n}-q\right\| \\
& \leq \eta_{n}\left\|g\left(x_{n}\right)-g(q)\right\|+\eta_{n}\|g(q)-q\|+\left(1-\eta_{n}\right)\left\|v_{n}-q\right\| \\
& \leq \eta_{n} \alpha\left\|x_{n}-q\right\|+\eta_{n}\|g(q)-q\|+\left(1-\eta_{n}\right)\left\|v_{n}-q\right\| .
\end{aligned}
$$

Using (30) and (31) in the above inequality, we have

$$
\begin{aligned}
\left\|x_{n+1}-q\right\| & \leq\left(1-(1-\alpha) \eta_{n}\right)\left\|x_{n}-q\right\|+\eta_{n} N_{1}+\eta_{n}\|g(q)-q\| \\
& =\left(1-(1-\alpha) \eta_{n}\right)\left\|x_{n}-q\right\|+\eta_{n} N_{1}+(1-\alpha) \eta_{n} \frac{N_{1}+\|g(q)-q\|}{1-\alpha} \\
& \leq \max \left\|x_{n}-q\right\|, \frac{N_{1}+\|g(q)-q\|}{1-\alpha} \leq \cdots \leq \max \left\|x_{n}-q\right\|, \frac{N_{1}+\|g(q)-q\|}{1-\alpha} .
\end{aligned}
$$


Initialization: choose $x_{0}, x_{1} \in H_{1}$ to be arbitrary.

Iterative Steps: given the current iterate $x_{n}$, compute:

Step 1. Compute $t_{n}=x_{n}-\theta_{n}\left(x_{n-1}-x_{n}\right)$

Step 2. Compute $u_{n}=T_{r_{n}}^{\left(G_{1}, b_{1}\right)}\left(t_{n}+\lambda_{n} B^{*}\left(T_{r_{n}}^{\left(G_{2}, b_{2}\right)}-I\right) B t_{n}\right)$

Step 3. Compute $v_{n}=P_{C}\left(u_{n}-\delta_{n} D u_{n}\right)$

and calculate the next iterate $x_{n+1}$ as follows:

$x_{n+1}=\eta_{n} g\left(x_{n}\right)+\left(1-\eta_{n}\right) v_{n}$

Set $n:=n+1$ and go to Step 1 .

Algorithm 1: Iterative algorithm.

Thus, $\left\{x_{n}\right\}$ is bounded. Also, $\left\{u_{n}\right\},\left\{v_{n}\right\}$, and $\left\{t_{n}\right\}$ are bounded.
Step 2. We show that $\left(1-\eta_{n}\right) \lambda_{n}\left(1-\lambda_{n} L\right) \|\left(T_{r_{n}}^{\left(G_{2}\right.}, b_{2}\right)-$ I) $B t_{n}\|\leq\| x_{n}-q\left\|^{2}-\right\| x_{n+1}-q \|^{2}+\eta_{n} N_{4}$, for some $N_{4}>0$. We estimate

$$
\begin{aligned}
\left\|x_{n+1}-q\right\|^{2} & \leq \eta_{n}\left\|g\left(x_{n}\right)-q\right\|^{2}+\left(1-\eta_{n}\right)\left\|v_{n}-q\right\|^{2} \\
& \leq \eta_{n}\left(\left\|g\left(x_{n}\right)-g(q)\right\|+\|g(q)-q\|\right)^{2}+\left(1-\eta_{n}\right)\left\|v_{n}-q\right\|^{2} \\
& \leq \eta_{n}\left(\alpha\left\|x_{n}-q\right\|+\|g(q)-q\|\right)^{2}+\left(1-\eta_{n}\right)\left\|v_{n}-q\right\|^{2} \\
& \leq \eta_{n}\left(\left\|x_{n}-q\right\|+\|g(q)-q\|\right)^{2}+\left(1-\eta_{n}\right)\left\|v_{n}-q\right\|^{2} \\
& =\eta_{n}\left\|x_{n}-q\right\|^{2}+\eta_{n}\left(2\left\|x_{n}-q\right\|\|g(q)-q\|+\|g(q)-q\|^{2}\right)+\left(1-\eta_{n}\right)\left\|v_{n}-q\right\|^{2} \\
& \leq \eta_{n}\left\|x_{n}-q\right\|^{2}+\left(1-\eta_{n}\right)\left\|u_{n}-q\right\|^{2}+\eta_{n} N_{2}, \quad \text { for some } N_{2}>0 .
\end{aligned}
$$

Using (26) in the above inequality, we get

$\left\|x_{n+1}-q\right\|^{2} \leq \eta_{n}\left\|x_{n}-q\right\|^{2}+\left(1-\eta_{n}\right)\left\|t_{n}-q\right\|^{2}$

$$
-\left(1-\eta_{n}\right) \lambda_{n}\left(1-\lambda_{n} L\right)\left\|\left(T_{r_{n}}^{\left(G_{2}, b_{2}\right)}-I\right) B t_{n}\right\|^{2}+\eta_{n} N_{2} \text {. }
$$

From (30), we obtain

$$
\begin{aligned}
\left\|t_{n}-q\right\|^{2} & \leq\left(\left\|x_{n}-q\right\|+\eta_{n} N_{1}\right)^{2} \\
& =\left\|x_{n}-q\right\|^{2}+\eta_{n}\left(2 N_{1}\left\|x_{n}-q\right\|+\eta_{n} N_{1}^{2}\right) \\
& \leq\left\|x_{n}-q\right\|^{2}+\eta_{n} N_{3}, \quad \text { for some } N_{3}>0 .
\end{aligned}
$$

By (35) and (36), we have

$$
\begin{aligned}
\left\|x_{n+1}-q\right\|^{2} \leq & \eta_{n}\left\|x_{n}-q\right\|^{2}+\left(1-\eta_{n}\right)\left\|x_{n}-q\right\|^{2}+\eta_{n} N_{3} \\
& \quad-\left(1-\eta_{n}\right) \lambda_{n}\left(1-\lambda_{n} L\right)\left\|\left(T_{r_{n}}^{\left(G_{2}, b_{2}\right)}-I\right) B t_{n}\right\|^{2}+\eta_{n} N_{2} \\
= & \left\|x_{n}-q\right\|^{2}+\eta_{n} N_{3}-\left(1-\eta_{n}\right) \lambda_{n}\left(1-\lambda_{n} L\right)\left\|\left(T_{r_{n}}^{\left(G_{2}, b_{2}\right)}-I\right) B t_{n}\right\|^{2}+\eta_{n} N_{2},
\end{aligned}
$$

which yields that

$$
\begin{aligned}
& \left(1-\eta_{n}\right) \lambda_{n}\left(1-\lambda_{n} L\right)\left\|\left(T_{r_{n}}^{\left(G_{2}, b_{2}\right)}-I\right) B t_{n}\right\|^{2} \\
& \leq\left\|x_{n+1}-q\right\|^{2}-\left\|x_{n}-q\right\|^{2}+\eta_{n} N_{4},
\end{aligned}
$$

where $N_{4}=N_{2}+N_{3}$.

Step 3. We show that

$$
\begin{aligned}
\left(1-\eta_{n}\right)\left\|u_{n}-t_{n}\right\|^{2} \leq & \left\|x_{n}-q\right\|^{2}-\left\|x_{n+1}-q\right\|^{2}+\eta_{n} N_{4} \\
& +2\left(1-\eta_{n}\right) \lambda_{n}\left\|u_{n}-q\right\|\left\|B^{*}\left(T_{r_{n}}^{\left(G_{2}, b_{2}\right)}-I\right) B t_{n}\right\| .
\end{aligned}
$$


Using the concept of firmly nonexpansive of $T_{r_{n}}^{\left(G_{2}, b_{2}\right)}$, we have

$$
\begin{aligned}
\left\|u_{n}-q\right\|^{2}= & \left\|T_{r_{n}}^{\left(G_{1}, \phi_{1}\right)}\left(t_{n}+\lambda_{n} B^{*}\left(T_{r_{n}}^{\left(G_{2}, \phi_{2}\right)}-I\right) B t_{n}\right)-q\right\|^{2} \\
= & \| T_{r_{n}}^{\left(G_{1}, \phi_{1}\right)}\left(t_{n}+\lambda_{n} B^{*}\left(T_{r_{n}}^{\left(G_{2}, \phi_{2}\right)}-I\right) B t_{n}-T_{r_{n}}^{\left(G_{1}, \phi_{1}\right)} q \|^{2}\right. \\
\leq & \left\langle u_{n}-q, t_{n}+\lambda_{n} B^{*}\left(T_{r_{n}}^{\left(G_{2}, \phi_{2}\right)}-I\right) B t_{n}-q\right\rangle \\
= & \frac{1}{2}\left\|u_{n}-q\right\|^{2}+\frac{1}{2}\left\|t_{n}+\lambda_{n} B^{*}\left(T_{r_{n}}^{\left(G_{2}, \phi_{2}\right)}-I\right) B t_{n}-q\right\|^{2} \\
& -\frac{1}{2}\left\|u_{n}-q-t_{n}-\lambda_{n} B^{*}\left(T_{r_{n}}^{\left(G_{2}, \phi_{2}\right)}-I\right) B t_{n}+q\right\|^{2} \\
= & \frac{1}{2}\left\|u_{n}-q\right\|^{2}+\frac{1}{2}\left\|t_{n}-q+\lambda_{n} B^{*}\left(T_{r_{n}}^{\left(G_{2}, \phi_{2}\right)}-I\right) B t_{n}\right\|^{2} \\
& -\frac{1}{2}\left\|u_{n}-t_{n}-\lambda_{n} B^{*}\left(T_{r_{n}}^{\left(G_{2}, \phi_{2}\right)}-I\right) B t_{n}\right\|^{2} \\
= & \frac{1}{2}\left\|u_{n}-q\right\|^{2}+\frac{1}{2}\left\|t_{n}-q\right\|^{2}+\frac{1}{2} \lambda_{n}^{2}\left\|B^{*}\left(T_{r_{n}}^{\left(G_{2}, \phi_{2}\right)}-I\right) B t_{n}\right\|^{2} \\
& +\left\langle t_{n}-q, \lambda_{n} B^{*}\left(T_{r_{n}}^{\left(G_{2}, \phi_{2}\right)}-I\right) B t_{n}\right\rangle-\frac{1}{2}\left\|u_{n}-t_{n}\right\|^{2} \\
& -\frac{1}{2} \lambda_{n}^{2}\left\|B^{*}\left(T_{r_{n}}^{\left(G_{2}, \phi_{2}\right)}-I\right) B t_{n}\right\|^{2}+\left\langle u_{n}-t_{n}, \lambda_{n} B^{*}\left(T_{r_{n}}^{\left(G_{2}, \phi_{2}\right)}-I\right) B t_{n}\right\rangle \\
= & \frac{1}{2}\left\|u_{n}-q\right\|^{2}+\frac{1}{2}\left\|t_{n}-q\right\|^{2}-\frac{1}{2}\left\|u_{n}-t_{n}\right\|^{2}+\left\langle u_{n}-q, \lambda_{n} B^{*}\left(T_{r_{n}}^{\left(G_{2}, \phi_{2}\right)}-I\right) B t_{n}\right\rangle,
\end{aligned}
$$

which implies that

$$
\begin{aligned}
\left\|u_{n}-q\right\|^{2} & \leq\left\|t_{n}-q\right\|^{2}-\left\|u_{n}-t_{n}\right\|^{2}+2\left\langle u_{n}-q, \mu_{n} B^{*}\left(T_{r_{n}}^{\left(G_{2}, \phi_{2}\right)}-I\right) B t_{n}\right\rangle \\
& \leq\left\|t_{n}-q\right\|^{2}-\left\|u_{n}-t_{n}\right\|^{2}+2 \mu_{n}\left\|u_{n}-q\right\|\left\|B^{*}\left(T_{r_{n}}^{\left(G_{2}, \phi_{2}\right)}-I\right) B t_{n}\right\| .
\end{aligned}
$$

Using (41) in (35), we get

$$
\begin{aligned}
\left\|x_{n+1}-q\right\|^{2} \leq & \eta_{n}\left\|x_{n}-q\right\|^{2}+\left(1-\eta_{n}\right)\left\|t_{n}-q\right\|^{2}-\left(1-\eta_{n}\right)\left\|u_{n}-t_{n}\right\|^{2} \\
& +2 \mu_{n}\left(1-\eta_{n}\right)\left\|u_{n}-q\right\|\left\|B^{*}\left(T_{r_{n}}^{\left(G_{2}, \phi_{2}\right)}-I\right) B t_{n}\right\|+\eta_{n} N_{2} .
\end{aligned}
$$


Using (36), in the above inequality,

$$
\begin{aligned}
\left\|x_{n+1}-q\right\|^{2} \leq & \eta_{n}\left\|x_{n}-q\right\|^{2}+\left(1-\eta_{n}\right)\left\|x_{n}-q\right\|^{2}+\eta_{n} N_{3}-\left(1-\eta_{n}\right)\left\|u_{n}-t_{n}\right\|^{2} \\
& +2 \mu_{n}\left(1-\eta_{n}\right)\left\|u_{n}-q\right\|\left\|B^{*}\left(T_{r_{n}}^{\left(G_{2}, \phi_{2}\right)}-I\right) B t_{n}\right\| \\
\leq & \left\|x_{n}-q\right\|^{2}-\left(1-\eta_{n}\right)\left\|u_{n}-t_{n}\right\|^{2} \\
& +2 \mu_{n}\left(1-\eta_{n}\right)\left\|u_{n}-q\right\|\left\|B^{*}\left(T_{r_{n}}^{\left(G_{2}, \phi_{2}\right)}-I\right) B t_{n}\right\|+\eta_{n} N_{4}
\end{aligned}
$$

which implies

$$
\begin{aligned}
\left(1-\eta_{n}\right)\left\|u_{n}-t_{n}\right\|^{2} \leq & \left\|x_{n}-q\right\|^{2}-\left\|x_{n+1}-q\right\|^{2} \\
& +2 \mu_{n}\left(1-\eta_{n}\right)\left\|u_{n}-q\right\| \\
& \left\|B^{*}\left(T_{r_{n}}^{\left(G_{2}, \phi_{2}\right)}-I\right) B t_{n}\right\|+\eta_{n} N_{4} .
\end{aligned}
$$

$$
\begin{aligned}
\left\|x_{n+1}-q\right\|^{2} \leq & \left(1-(1-\alpha) \eta_{n}\right)\left\|x_{n}-q\right\|^{2}+(1-\alpha) \eta_{n} \\
& \times\left[\frac{2}{1-\alpha}\left\langle g(q)-q, x_{n+1}-q\right\rangle+\frac{\theta_{n}}{\eta_{n}}\left\|x_{n}-x_{n-1}\right\| \frac{N}{1-\alpha}\right],
\end{aligned}
$$

for some $N>0$.

We estimate

$$
\begin{aligned}
\left\|t_{n}-q\right\|^{2} & =\left\|x_{n}+\theta_{n}\left(x_{n}-x_{n-1}\right)-q\right\|^{2} \\
& =\left\|x_{n}-q\right\|^{2}+2 \theta_{n}\left\langle x_{n}-q, x_{n}-x_{n-1}\right\rangle+\theta_{n}^{2}\left\|x_{n}-x_{n-1}\right\|^{2} \\
& \leq\left\|x_{n}-q\right\|^{2}+2 \theta_{n}\left\|x_{n}-q\right\|\left\|x_{n}-x_{n-1}\right\|+\theta_{n}^{2}\left\|x_{n}-x_{n-1}\right\|^{2} \\
& =\left\|x_{n}-q\right\|^{2}+\theta_{n}\left\|x_{n}-x_{n-1}\right\|\left(2\left\|x_{n}-q\right\|+\theta_{n}\left\|x_{n}-x_{n-1}\right\|\right) \\
& \leq\left\|x_{n}-q\right\|^{2}+\theta_{n}\left\|x_{n}-x_{n-1}\right\| N, \quad \text { for some } N>0 .
\end{aligned}
$$

Using (14), we calculate

$$
\begin{aligned}
\left\|x_{n+1}-q\right\|^{2} & =\left\|\eta_{n} g\left(x_{n}\right)+\left(1-\eta_{n}\right) u_{n}-q\right\|^{2} \\
& =\left\|\eta_{n}\left(g\left(x_{n}\right)-g(q)\right)+\left(1-\eta_{n}\right)\left(u_{n}-q\right)+\eta_{n}(g(q)-q)\right\|^{2} \\
& \leq\left\|\eta_{n}\left(g\left(x_{n}\right)-g(q)\right)+\left(1-\eta_{n}\right)\left(u_{n}-q\right)\right\|^{2}+2 \eta_{n}\left\langle g(q)-q, x_{n+1}-q\right\rangle \\
& \leq \eta_{n}\left\|g\left(x_{n}\right)-g(q)\right\|^{2}+\left(1-\eta_{n}\right)\left\|u_{n}-q\right\|^{2}+2 \eta_{n}\left\langle g(q)-q, x_{n+1}-q\right\rangle \\
& \leq \eta_{n} \alpha\left\|x_{n}-q\right\|^{2}+\left(1-\eta_{n}\right)\left\|u_{n}-q\right\|^{2}+2 \eta_{n}\left\langle g(q)-q, x_{n+1}-q\right\rangle \\
& \leq \eta_{n} \alpha\left\|x_{n}-q\right\|^{2}+\left(1-\eta_{n}\right)\left\|t_{n}-q\right\|^{2}+2 \eta_{n}\left\langle g(q)-q, x_{n+1}-q\right\rangle .
\end{aligned}
$$


From (46) and (47), we have

$$
\begin{aligned}
\left\|x_{n+1}-q\right\|^{2} \leq & \left(1-(1-\alpha) \eta_{n}\right)\left\|x_{n}-q\right\|^{2}+\theta_{n}\left\|x_{n}-x_{n-1}\right\| N+2 \eta_{n}\left\langle g(q)-q, x_{n+1}-q\right\rangle \\
= & \left(1-(1-\alpha) \eta_{n}\right)\left\|x_{n}-q\right\|^{2}+(1-\alpha) \eta_{n} \\
& \times\left[\frac{2}{1-\alpha}\left\langle g(q)-q, x_{n+1}-q\right\rangle+\frac{\theta_{n}}{\eta_{n}}\left\|x_{n}-x_{n-1}\right\| \frac{N}{1-\alpha}\right] .
\end{aligned}
$$

Step 5. We show that $\lim _{n \longrightarrow \infty}\left\|x_{n}-q\right\|=0$.

To show it, we have the two cases as follows:

Case 1. There exists $m \in \mathbb{N}$ such that $\left\|x_{n+1}-q\right\|^{2} \leq\left\|x_{n}-q\right\|^{2}, \forall n \geq m$. This shows that $\lim _{n \rightarrow \infty}\left\|x_{n}-q\right\|$ exists, and by step 2, we have

$$
\left.\lim _{n \longrightarrow \infty} \|\left(T_{r_{n}}^{\left(G_{2}, b_{2}\right)}-I\right) B t_{n}\right) \|=0 .
$$

$$
\lim _{n \longrightarrow \infty}\left\|x_{n+1}-v_{n}\right\|=0
$$

Now,

$$
\begin{array}{r}
\left\|x_{n}-t_{n}\right\|=\theta_{n}\left\|x_{n}-x_{n-1}\right\|=\frac{\theta_{n}}{\eta_{n}}\left\|x_{n}-x_{n-1}\right\| \eta_{n} \longrightarrow 0, \\
\text { as } n \longrightarrow \infty .
\end{array}
$$

Thanks to step 3 and (49), we obtain

$$
\lim _{n \longrightarrow \infty}\left\|u_{n}-t_{n}\right\|=0 \text {. }
$$

Next, prove that $\lim _{n \longrightarrow \infty}\left\|u_{n}-v_{n}\right\|=0$.

By (47), we have

Since $\left\|x_{n+1}-u_{n}\right\|=\eta_{n}\left\|u_{n}-g\left(x_{n}\right)\right\|$, therefore

$$
\begin{aligned}
\left\|x_{n+1}-q\right\|^{2} & \leq \eta_{n} \alpha\left\|x_{n}-q\right\|^{2}+\left(1-\eta_{n}\right)\left\|v_{n}-q\right\|^{2}+2 \eta_{n}\left\langle g(q)-q, x_{n+1}-q\right\rangle \\
& =\eta_{n} \alpha\left\|x_{n}-q\right\|^{2}+\left(1-\eta_{n}\right)\left\|v_{n}-q\right\|^{2}+2 \eta_{n}\left\langle\zeta, x_{n+1}-q\right\rangle \\
& \leq \eta_{n} \alpha\left\|x_{n}-q\right\|^{2}+\left(1-\eta_{n}\right)\left\|v_{n}-q\right\|^{2}+2 \eta_{n} \varrho^{2} .
\end{aligned}
$$

We set $\zeta=f(q)-q$ and let $\varrho>0$ be a suitable constant with $\varrho \geq \sup _{n}\left\{\|\zeta\|,\left\|x_{n}-q\right\|\right\}$ in the above inequality.

Thus,

$$
\begin{aligned}
\left\|x_{n+1}-q\right\|^{2} & \leq\left(1-\eta_{n}\right)\left\{\left\|P_{C}\left(u_{n}-\lambda_{n} B u_{n}\right)-P_{C}\left(q-\lambda_{n} B q\right)\right\|^{2}\right\}+\eta_{n}\left\|x_{n}-q\right\|^{2}+2 \eta_{n} \varrho^{2} \\
& \leq\left(1-\eta_{n}\right)\left\{\left\|u_{n}-q\right\|^{2}+\lambda_{n}\left(\lambda_{n}-2 \gamma\right)\left\|B u_{n}-B q\right\|^{2}\right\}+\eta_{n}\left\|x_{n}-q\right\|^{2}+2 \eta_{n} \varrho^{2} \\
& \leq\left(1-\eta_{n}\right)\left\{\left\|x_{n}-\tilde{x}\right\|^{2}+\lambda_{n}\left(\lambda_{n}-2 \gamma\right)\left\|B u_{n}-B q\right\|^{2}\right\}+\eta_{n}\left\|x_{n}-\tilde{x}\right\|^{2}+2 \eta_{n} \varrho^{2} \\
& \leq\left(1-\eta_{n}\right) \lambda_{n}\left(\lambda_{n}-2 \gamma\right)\left\|B u_{n}-B q\right\|^{2}+\left\|x_{n}-q\right\|^{2}+2 \eta_{n} \varrho^{2} .
\end{aligned}
$$


This implies

$$
\begin{aligned}
& \left(1-\eta_{n}\right) \lambda_{n}\left(2 \gamma-\lambda_{n}\right)\left\|B u_{n}-B q\right\|^{2} \\
& \leq\left\|x_{n}-q\right\|^{2}-\left\|x_{n+1}-q\right\|^{2}+2 \eta_{n} \varrho^{2} .
\end{aligned}
$$

Thus,

$$
\lim _{n \longrightarrow \infty}\left\|B u_{n}-B q\right\|=0 .
$$

We compute

$$
\begin{aligned}
\left\|v_{n}-q\right\|^{2} & =\left\|P_{C}\left(u_{n}-\lambda_{n} B u_{n}\right)-P_{C}\left(q-\lambda_{n} B q\right)\right\|^{2} \\
& \leq\left\langle v_{n}-q,\left(u_{n}-\lambda_{n} B u_{n}\right)-\left(q-\lambda_{n} B q\right)\right\rangle \\
& \leq \frac{1}{2}\left\{\left\|v_{n}-q\right\|^{2}+\left\|\left(u_{n}-\lambda_{n} B u_{n}\right)-\left(q-\lambda_{n} B q\right)\right\|^{2}-\left\|\left(v_{n}-u_{n}\right)+\lambda_{n}\left(B u_{n}-B q\right)\right\|^{2}\right\} \\
& \leq \frac{1}{2}\left\{\left\|v_{n}-q\right\|^{2}+\left\|u_{n}-q\right\|^{2}-\left\|\left(v_{n}-u_{n}\right)+\lambda_{n}\left(B u_{n}-B q\right)\right\|^{2}\right\} \\
& \leq\left\|u_{n}-q\right\|^{2}-\left\|v_{n}-u_{n}\right\|^{2}-\lambda_{n}^{2}\left\|B u_{n}-B q\right\|^{2}+2 \lambda_{n}\left\langle v_{n}-u_{n}, B v_{n}-B q\right\rangle \\
& \leq\left\|u_{n}-q\right\|^{2}-\left\|v_{n}-u_{n}\right\|^{2}+2 \lambda_{n}\left\|v_{n}-u_{n}\right\|\left\|B u_{n}-A q\right\| \\
& \leq\left\|x_{n}-q\right\|^{2}-\left\|v_{n}-u_{n}\right\|^{2}+2 \lambda_{n}\left\|v_{n}-u_{n}\right\|\left\|B u_{n}-A q\right\| .
\end{aligned}
$$

By (53), we get

$$
\begin{aligned}
\left\|x_{n+1}-q\right\|^{2} & \leq\left(1-\lambda_{n}\right)\left\|v_{n}-q\right\|^{2}+\lambda_{n}\left\|x_{n}-q\right\|^{2}+2 \eta_{n} \varrho^{2} \\
& \leq\left(1-\lambda_{n}\right)\left\{\left\|x_{n}-q\right\|^{2}-\left\|v_{n}-u_{n}\right\|^{2}+2 \lambda_{n}\left\|v_{n}-u_{n}\right\|\left\|B u_{n}-B q\right\|\right\}+\lambda_{n}\left\|x_{n}-q\right\|^{2}+2 \eta_{n} \varrho^{2},
\end{aligned}
$$

which implies

$$
\left(1-\lambda_{n}\right)\left\|v_{n}-u_{n}\right\|^{2} \leq\left\|x_{n}-q\right\|^{2}-\left\|x_{n+1}-q\right\|^{2}+2\left(1-\lambda_{n}\right) \lambda_{n}\left\|v_{n}-u_{n}\right\|\left\|B u_{n}-B q\right\|+2 \eta_{n} \varrho^{2} .
$$

Using (56) and the given conditions, we get

$$
\lim _{n \longrightarrow \infty}\left\|v_{n}-u_{n}\right\|=0 \text {. }
$$

From (50)-(52) and (60), we have

$$
\begin{array}{r}
\left\|x_{n+1}-x_{n}\right\| \leq\left\|x_{n+1}-u_{n}\right\|+\left\|u_{n}-t_{n}\right\|+\left\|t_{n}-x_{n}\right\| \\
\text { as } n \longrightarrow 0,
\end{array}
$$

We prove that $\limsup _{n \rightarrow \infty}\left\langle(g-I) q, x_{n}-q\right\rangle \leq 0$.
Since $\left\{u_{n}\right\}$ is bounded, there exists a subsequence $\left\{u_{n_{i}}\right\}$ of $\left\{u_{n}\right\}$ which converges weakly to some $p \in C$. Without loss of generality, we can assume that $u_{n_{i}} \rightarrow p$ such that

$$
\underset{n \longrightarrow \infty}{\limsup }\left\langle(g-I) q, u_{n}-q\right\rangle=\lim _{i \longrightarrow \infty}\left\langle(g-I) q, u_{n_{i}}-q\right\rangle .
$$

We prove that $p \in \Gamma \cap \Omega$.

Since $u_{n}=T_{r_{n}}^{\left(G_{1}, b_{1}\right)} d_{n}$ where $d_{n}:=t_{n}+\lambda_{n} B^{*}\left(T_{r_{n}}^{\left(G_{2}, b_{2}\right)}-I\right)$ $B t_{n}$, we have

$$
G_{1}\left(u_{n}, u\right)+b_{1}\left(u, u_{n}\right)-b_{1}\left(u_{n}, u_{n}\right)+\frac{1}{r_{n}}\left\langle u-u_{n}, u_{n}-d_{n}\right\rangle \geq 0, \quad \forall u \in C,
$$


which implies that

$b_{1}\left(u, u_{n}\right)-b_{1}\left(u_{n}, u_{n}\right)+\frac{1}{r_{n}}\left\langle u-u_{n}, u_{n}-d_{n}\right\rangle \geq G_{1}\left(u, u_{n}\right)$,

$\forall u \in C$ (using monotonocity of $\left.G_{1}\right)$.

Hence,

$b_{1}\left(u, u_{n_{k}}\right)-b_{1}\left(u_{n_{k}}, u_{n_{k}}\right)+\left\langle u-u_{n_{k}}, \frac{u_{n_{k}}-d_{n_{k}}}{r_{n_{k}}}\right\rangle \geq G_{1}\left(u, u_{n_{k}}\right)$,

$\forall u \in C$.

Let $u_{t}=(1-t) w+t u$, for all $t \in(0,1]$. Since $u \in C$ and $w \in C$, we get $u_{t} \in C$ and from (65), we have

$0 \leq G_{1}\left(u_{t}, u_{n_{k}}\right)-b_{1}\left(u_{t}, u_{n_{k}}\right)+b_{1}\left(u_{n_{k}}, u_{n_{k}}\right)$

$$
-\left\langle u_{t}-u_{n_{k}}, \frac{u_{n_{k}}-t_{n_{k}}}{r_{n_{k}}}+\lambda_{n} B^{*}\left(\frac{\left(T_{r_{n_{k}}}^{\left(G_{2}, b_{2}\right)}-I\right) B t_{n_{k}}}{r_{n_{k}}}\right)\right\rangle .
$$

Since $B^{*}$ is bounded linear, it follows from (49), (50), (60), and $\liminf r_{n}>0$ that $\left(\left(u_{n_{k}}-t_{n_{k}}\right) / r_{n_{k}}\right) \longrightarrow 0$ and $B^{*}\left(\left(T_{r_{n_{k}}}^{\left(G_{2}, b_{2}\right)}-I\right) B t_{n_{k}} / r_{n_{k}}\right) \longrightarrow 0$ and so

$$
b_{1}\left(u_{t}, p\right)-b_{1}(p, p) \leq G_{1}\left(u_{t}, p\right) \text {. }
$$

Now, for $t>0$,

$$
\begin{aligned}
0 & =G_{1}\left(u_{t}, u_{t}\right) \\
& =t G_{1}\left(u_{t}, u_{t}\right)+(1-t) G_{1}\left(u_{t}, p\right) \\
& \geq t G_{1}\left(u_{t}, u_{t}\right)+(1-t)\left[b_{1}\left(u_{t}, p\right)-b_{1}(p, p)\right] \\
& \geq t G_{1}\left(u_{t}, u_{t}\right)+(1-t) t\left[b_{1}(u, p)-b_{1}(p, p)\right] \\
& \geq G_{1}\left(u_{t}, u_{t}\right)+(1-t)\left[b_{1}(u, p)-b_{1}(p, p)\right] .
\end{aligned}
$$

Letting $t \longrightarrow 0$, we have

$$
G_{1}(p, u)+b_{1}(u, p)-b_{1}(p, p) \geq 0, \quad \forall u \in C .
$$

This implies that $p \in \operatorname{Sol}(\operatorname{GEP}(4))$.

Next, we show that $B p \in \operatorname{Sol}(\operatorname{GEP}(5))$. Since $\left\|u_{n}-t_{n}\right\| \longrightarrow 0, u_{n} \rightarrow p$ as $n \longrightarrow \infty$ and $\left\{t_{n}\right\}$ is bounded, there exists a subsequence $\left\{t_{n_{k}}\right\}$ of $\left\{t_{n}\right\}$ such that $t_{n_{k}} \rightarrow p$ and since $B$ is a bounded linear operator so that $B t_{n_{k}} \rightarrow B p$.

Now, setting $v_{n_{k}}=B t_{n_{k}}-T_{r_{n_{k}}}^{\left(G_{2}, b_{2}\right)} B t_{n_{k}}$, it follows that from (49), $\lim _{k \longrightarrow \infty} v_{n_{k}}=0$ and $B t_{n_{k}}-v_{n_{k}}=T_{r_{n_{k}}}^{\left(G_{2}, b_{2}\right)} B t_{n_{k}}$. Therefore, from Lemma 5, we have

$$
\begin{aligned}
& G_{2}\left(B t_{n_{k}}-v_{n_{k}}, z\right)+b_{1}\left(z, u_{n_{k}}\right)-b_{1}\left(u_{n_{k}}, u_{n_{k}}\right) \\
& \quad+\frac{1}{r_{n_{k}}}\left\langle z-\left(B t_{n_{k}}-v_{n_{k}}\right),\left(B t_{n_{k}}-v_{n_{k}}\right)-B t_{n_{k}}\right\rangle \geq 0, \quad \forall z \in Q .
\end{aligned}
$$

Since $G_{2}$ is upper semicontinuous in the first argument, taking limit superior to the above inequality as $k \longrightarrow \infty$ and using condition, we obtain

$$
G_{2}(B p, z)+b_{1}\left(z, u_{n_{k}}\right)-b_{1}\left(u_{n_{k}}, u_{n_{k}}\right) \geq 0, \quad \forall z \in Q,
$$

which means that $B p \in \operatorname{Sol}(\operatorname{GEP}(5))$ and hence $p \in \Gamma$. Next, we prove $p \in \Omega$. Since $\lim _{n \longrightarrow \infty}\left\|u_{n}-v_{n}\right\|=0$ and $\lim _{n \longrightarrow \infty}\left\|u_{n}-t_{n}\right\|=0$, there exist subsequences $\left\{u_{n_{i}}\right\}$ and $\left\{v_{n_{i}}\right\}$ of $\left\{u_{n}\right\}$ and $\left\{v_{n}\right\}$, respectively such that $u_{n_{i}} \rightarrow p$ and $v_{n_{i}} \rightarrow p$.

Define the mapping $M$ as

$$
M(z)= \begin{cases}D(z)+N_{C}(p), & \text { if } p \in C, \\ \varnothing, & \text { if } p \notin C,\end{cases}
$$

where $N_{C}(p):=\left\{v \in H_{1}:\langle p-u, v\rangle \geq 0, \forall u \in C\right\}$ is the normal cone to $C$ at $p \in H_{1}$. In this case, the mapping $M$ is maximal monotone and hence $0 \in M p$ mapping if and only if $p \in \operatorname{Sol}(\operatorname{VIP}(1))$. Let $(p, v) \in \operatorname{graph}(M)$. Then, we have $v \in M p=D p+N_{C}(p)$ and hence $v-D p \in N_{C}(p)$. So, we have $\langle p-u, v-D p\rangle \geq 0$, for all $u \in C$. On the other hand, from $v_{n}=P_{C}\left(u_{n}-\delta_{n} D u_{n}\right)$ and $p \in C$, we have

$$
\left\langle\left(u_{n}-\delta_{n} D u_{n}\right)-v_{n}, v_{n}-p\right\rangle \geq 0 .
$$

This implies that

$$
\left\langle z-v_{n}, \frac{v_{n}-u_{n}}{\delta_{n}}+D u_{n}\right\rangle \geq 0
$$

Since $\langle z-u, v-D z\rangle \geq 0$, for all $z \in C$ and $v_{n_{i}} \in C$, using monotonicity of $D$, we have 


$$
\begin{aligned}
\left\langle z-v_{n_{i}}, v\right\rangle & \geq\left\langle z-v_{n_{i}}, D p\right\rangle \\
& \geq\left\langle z-v_{n_{i}}, D z\right\rangle-\left\langle z-v_{n_{i}}, \frac{v_{n_{i}}-u_{n_{i}}}{\delta_{n}}+D u_{n_{i}}\right\rangle \\
& =\left\langle z-v_{n_{i}}, D z-D u_{n_{i}}\right\rangle+\left\langle z-v_{n_{i}}, D v_{n_{i}}-D u_{n_{i}}\right\rangle-\left\langle z-v_{n_{i}}, \frac{v_{n_{i}}-u_{n_{i}}}{\delta_{n}}\right\rangle \\
& \geq\left\langle z-v_{n_{i}}, D v_{n_{i}}-D u_{n_{i}}\right\rangle\left\langle\left\langle z-v_{n_{i}}, \frac{v_{n_{i}}-u_{n_{i}}}{\delta_{n}}\right\rangle .\right.
\end{aligned}
$$

Since $D$ is continuous therefore on taking limit $i \longrightarrow \infty$, we have $\langle z-p, v\rangle \geq 0$. Since $M$ is maximal monotone, we have $p \in M^{-1}(0)$ and hence $p \in \Omega$. Thus, $p \in \Gamma \cap \Omega$.

Since $q=P_{\Theta}(g) q$, therefore from (62),

$$
\limsup _{n \longrightarrow \infty}\left\langle(g-I) q, u_{n}-q\right\rangle=\lim _{i \longrightarrow \infty}\langle(g-I) q, p-q\rangle \leq 0 .
$$

Using Lemma 4, (76), and the given conditions in step 4 , we get $x_{n} \longrightarrow q$, where $q=P_{\Theta}(g) q$.

Case 2. There exists a subsequence $\left\{\left\|x_{n_{i}}-q\right\|^{2}\right\}$ of $\left\{\left\|x_{n}-q\right\|^{2}\right\}$ such that $\left\|x_{n_{i}}-q\right\|^{2}<\left\|x_{n_{i+1}}-q\right\|^{2}, \forall i \in \mathbb{N}$. Thus, by Lemma 2, $\exists$ is a nondecreasing sequence $m_{j}$ of $\mathbb{N}$ such that $\lim _{j \longrightarrow \infty} m_{j}=\infty$ and

$$
\begin{gathered}
\left\|x_{m_{j}}-q\right\|^{2} \leq\left\|x_{m_{j+1}}-q\right\|^{2}, \\
\left\|x_{j}-q\right\|^{2} \leq\left\|x_{m_{j}}-q\right\|^{2} .
\end{gathered}
$$

By step 2, we get

$$
\begin{aligned}
& \left.\left(1-\eta_{m_{j}}\right) \lambda_{m_{j}}\left(1-\lambda_{m_{j}} L\right) \|\left(T_{r_{m_{j}}}^{\left(G_{2}, b_{2}\right)}-I\right) B t_{m_{j}}\right) \| \\
& \leq\left\|x_{m_{j}}-q\right\|^{2}-\left\|x_{m_{j+1}}-q\right\|^{2}+\alpha_{m_{j}} N_{4} \leq \alpha_{m_{j}} N_{4} .
\end{aligned}
$$

Thus,

$$
\left.\lim _{n \longrightarrow \infty} \|\left(T_{r_{m_{j}}}^{\left(G_{2}, b_{2}\right)}-I\right) B t_{m_{j}}\right) \|=0 .
$$

By step 3, we obtain

$$
\begin{aligned}
\left(1-\eta_{m_{j}}\right)\left\|u_{m_{j}}-t_{m_{j}}\right\|^{2} \leq & \left\|x_{m_{j}}-q\right\|^{2}-\left\|x_{m_{j+1}}-q\right\|^{2}+\eta_{m_{j}} N_{4} \\
& +2\left(1-\eta_{m_{j}}\right) \lambda_{m_{j}}\left\|u_{m_{j}}-q\right\|\left\|B^{*}\left(T_{r_{m_{j}}}^{\left(G_{2}, b_{2}\right)}-I\right) B t_{m_{j}}\right\| \\
\leq & \eta_{m_{j}} N_{4}+2\left(1-\eta_{m_{j}}\right) \lambda_{m_{j}}\left\|u_{m_{j}}-q\right\|\left\|B^{*}\left(T_{r_{m_{j}}}^{\left(G_{2}, b_{2}\right)}-I\right) B t_{m_{j}}\right\| .
\end{aligned}
$$

Hence,

$$
\lim _{n \longrightarrow \infty}\left\|u_{m_{j}}-t_{m_{j}}\right\|=0 .
$$

By the similar steps of case 1 , we get

$$
\lim _{n \longrightarrow \infty}\left\|x_{m_{j+1}}-x_{m_{j}}\right\|=0,
$$

$$
\underset{j \longrightarrow \infty}{\limsup }\left\langle(g-I) q, x_{m_{j+1}}-q\right\rangle \leq 0 \text {. }
$$

By step 4, we have

$$
\begin{aligned}
\left\|x_{m_{j+1}}-q\right\|^{2} \leq & \left(1-(1-\alpha) \eta_{m_{j}}\right)\left\|x_{m_{j}}-q\right\|^{2}+(1-\alpha) \eta_{m_{j}} \\
& \times\left[\frac{2}{1-\alpha}\left\langle g(q)-q, x_{m_{j+1}}-q\right\rangle+\frac{\sigma_{m_{j}}}{\eta_{m_{j}}}\left\|x_{m_{j}}-x_{m_{j-1}}\right\| \frac{N}{1-\alpha}\right] .
\end{aligned}
$$


By (77) and (83), we have

$$
\begin{aligned}
\left\|x_{m_{j+1}}-q\right\|^{2} \leq & \left(1-(1-\alpha) \eta_{m_{j+1}}\right)\left\|x_{m_{j}}-q\right\|^{2}+(1-\alpha) \eta_{m_{j}} \\
& \times\left[\frac{2}{1-\alpha}\left\langle g(q)-q, x_{m_{j+1}}-q\right\rangle+\frac{\sigma_{m_{j}}}{\eta_{m_{j}}}\left\|x_{m_{j}}-x_{m_{j-1}}\right\| \frac{N}{1-\alpha}\right] .
\end{aligned}
$$

This implies

$$
\begin{gathered}
\left\|x_{m_{j+1}}-q\right\|^{2} \leq \frac{2}{1-\alpha}\left\langle g(q)-q, x_{m_{j+1}}-q\right\rangle+\frac{\sigma_{m_{j}}}{\eta_{m_{j}}} \\
\left\|x_{m_{j}}-x_{m_{j-1}}\right\| \frac{N}{1-\alpha} .
\end{gathered}
$$

Thus,

$$
\underset{j \longrightarrow \infty}{\limsup }\left\|x_{m_{j+1}}-q\right\|^{2} \leq 0 .
$$
proof.

By (77) and (86), $x_{j} \longrightarrow q$. This completes the

Moreover, we have the following consequences. If we take $\theta_{n}=0$, then Theorem 1 reduced to the following result without inertial as follows:

Corollary 1. Let $C$ and $Q$ be two nonempty closed convex subsets of Hilbert spaces $H_{1}$ and $H_{2}$, respectively. Let $B: H_{1} \longrightarrow H_{2}$ be a bounded linear operator. Assume that $G_{1}: C \times C \longrightarrow \mathbb{R}, G_{2}: Q \times Q \longrightarrow \mathbb{R}, b_{1}: C \times C \longrightarrow \mathbb{R}$, and $b_{2}: Q \times Q \longrightarrow \mathbb{R}$ are nonlinear mappings satisfying Assumption 1 and $G_{2}$ is upper semicontinuous in the first argument. Assume that $\Theta=\Gamma \cap \Omega \neq \varnothing$. Let $g: H_{1} \longrightarrow H_{1}$ be a contraction mapping with constant $\alpha \in(0,1)$ and $D: C \longrightarrow H_{1}$ be a $\tau$-inverse strongly monotone mapping. Let $\left\{x_{n}\right\}$ be generated by

$$
\left.\begin{array}{l}
x_{1} \in H_{1} \\
u_{n}=T_{r_{n}}^{\left(G_{1}, b_{1}\right)}\left(t_{n}+\lambda_{n} B^{*}\left(T_{r_{n}}^{\left(G_{2}, b_{2}\right)}-I\right) B t_{n}\right) \\
v_{n}=P_{C}\left(u_{n}-\delta_{n} D u_{n}\right) \\
x_{n+1}=\eta_{n} g\left(x_{n}\right)+\left(1-\eta_{n}\right) v_{n}
\end{array}\right\},
$$

where the control sequence satisfies the following conditions:

(i) $\lim _{n \longrightarrow \infty} \eta_{n}=0, \sum_{n=0}^{\infty} \eta_{n}=\infty$;

(ii) $\liminf _{n \longrightarrow \infty} r_{n}>0$ an $\lim _{n \longrightarrow \infty}\left|r_{n+1}-r_{n}\right|=0$;

(iii) $\left\{\lambda_{n}\right\} \subset \mathbb{R}$ such that $a \leq \lambda_{n} \leq b<(1 / L)$, where $L=\|B\|^{2}$.

Then, the sequence $\left\{x_{n}\right\}$ converges strongly to some $q \in \Theta$, where $q=P_{\Theta}(g) q$.

Further, if we take $b_{1}, b_{2} \equiv 0$, then Theorem 1 reduced to the following result as follows:
Corollary 2. Let $C$ and $Q$ be two nonempty closed convex subsets of Hilbert spaces $H_{1}$ and $H_{2}$, respectively. Let $B: H_{1} \longrightarrow H_{2}$ be a bounded linear operator. Assume that $G_{1}: C \times C \longrightarrow \mathbb{R}$ and $G_{2}: Q \times Q \longrightarrow \mathbb{R}$ are nonlinear mappings satisfying Assumption 1 (1)-(4) and $G_{2}$ is upper semicontinuous in first argument. Assume that $\Theta=\Upsilon \cap \Omega \neq \varnothing$, where $\Upsilon$ denotes the solution set of $S_{P} E P$ (6) and (7). Let $g: H_{1} \longrightarrow H_{1}$ be a contraction mapping with constant $\alpha \in(0,1)$ and $D: C \longrightarrow H_{1}$ be a $\tau$-inverse strongly monotone mapping. Let $\left\{x_{n}\right\}$ be generated by

$$
\left.\begin{array}{l}
x_{1} \in H_{1} \\
u_{n}=T_{r_{n}}^{\left(G_{1}\right)}\left(t_{n}+\lambda_{n} B^{*}\left(T_{r_{n}}^{\left(G_{2}\right)}-I\right) B t_{n}\right) \\
v_{n}=P_{C}\left(u_{n}-\delta_{n} D u_{n}\right) \\
x_{n+1}=\eta_{n} g\left(x_{n}\right)+\left(1-\eta_{n}\right) v_{n}
\end{array}\right\},
$$

where the control sequence satisfies the following conditions:

(i) $\lim _{n \longrightarrow \infty} \eta_{n}=0, \sum_{n=0}^{\infty} \eta_{n}=\infty$;

(ii) $\lim _{n \rightarrow \infty}\left(\theta_{n} / \eta_{n}\right)\left\|x_{n}-x_{n-1}\right\|=0$;

(iii) $\left\{\theta_{n}\right\} \subset[0, \theta]$, for some $\theta>0$ and $\delta_{n} \subset(0,2 \tau)$;

(iv) $\liminf _{n \longrightarrow \infty} r_{n}>0$ and $\lim _{n \longrightarrow \infty}\left|r_{n+1}-r_{n}\right|=0$;

(v) $\left\{\lambda_{n}\right\} \subset \mathbb{R}$ such that $a \leq \lambda_{n} \leq b<(1 / L)$, where $L=\|B\|^{2}$.

Then, the sequence $\left\{x_{n}\right\}$ converges strongly to some $q \in \Theta$, where $q=P_{\Theta}(g) q$.

\section{Numerical Illustration}

Finally, to supporting our main theorem, we now give an example in infinitely dimensional spaces $L_{2}[0,1]$ such that $\|\cdot\|$ is $L_{2}$-norm defined by $\|x\|=\sqrt{\int_{0}^{1}|x(t)|^{2} \mathrm{~d} t}$ where $x(t) \in L_{2}[0,1]$.

Example 1. Let $H_{1}=H_{2}=L_{2}[0,1]$ and $C=Q=\left\{x(t) \in L_{2}\right.$ $\left.[0,1]: \int_{0}^{1} t x(t) \mathrm{d} t \leq 1\right\}$. Define mappings as follows:

(i) bounded linear operator $B: H_{1} \longrightarrow H_{2}$ by $B x(t)=3 x(t), \forall x(t) \in L_{2}[0,1]$;

(ii) contraction mapping $g$ : $H_{1} \longrightarrow H_{1}$ by $g(x(t))=$ $\alpha x(t)$ where $\alpha \in[0,1)$;

(iii) nonlinear mappings $G_{1}: C \times C \longrightarrow \mathbb{R}$, $G_{2}: Q \times Q \longrightarrow \mathbb{R}$ by 
TABLE 1: Numerical results of $\bar{\theta}_{n}$.

\begin{tabular}{lccccc}
\hline $\bar{\theta}_{n}$ & 0 & 0.5 & $(1 / n)$ & $\left(1 / n^{2}\right)$ & $\min \left\{\left(1 / n^{2}\left\|x_{n}-x_{n-1}\right\|\right), 0.5\right\}$ \\
\hline No. of iters & 15 & 4 & 14 & 10 & 4 \\
CPU time (s) & 4.106713 & 1.862612 & 3.764422 & 2.922132 & 2.023393 \\
\hline
\end{tabular}

TABLE 2: Numerical results of $r_{n}$.

\begin{tabular}{lccccc}
\hline$r_{n}$ & 0.001 & 0.01 & 0.1 & 1 & 10 \\
\hline No. of iters & 15 & 14 & 4 & 3 & 5 \\
CPU time (s) & 4.169121 & 3.927234 & 1.914940 & 1.678531 & 2.077360 \\
\hline
\end{tabular}

TABLE 3: Numerical results of $\alpha_{n}$.

\begin{tabular}{lccccc}
\hline$\eta_{n}$ & $(1 /(n+1))$ & $(1 /(10 n+1))$ & $(1 /(20 n+1))$ & $(1 /(100 n+1))$ & $(1 / \sqrt{n})$ \\
\hline No. of ites. & 3 & 8 & 8 & 6 \\
CPU time (s) & 1.678531 & 2.710736 & 2.728162 & 2.700151 & 3.312465 \\
\hline
\end{tabular}

$$
\begin{array}{r}
G_{1}(x(t), y(t))=G_{2}(x(t), y(t))=\langle y(t)-x(t), x(t)\rangle, \\
\forall x(t), y(t) \in L_{2}[0,1],
\end{array}
$$

(iv) nonlinear mappings $b_{1}: C \times C \longrightarrow \mathbb{R}$ and $b_{2}: Q \times$ $Q \longrightarrow \mathbb{R}$ by

$$
\begin{array}{r}
b_{1}(x(t), y(t))=b_{2}(x(t), y(t))=\langle y(t), y(t)\rangle, \\
\forall x(t), y(t) \in L_{2}[0,1] .
\end{array}
$$

(v) $\tau$-inverse strongly monotone mapping $D: C \longrightarrow H_{1}$ by

$$
D x(t)=B^{*}\left(I-P_{Q}\right) B x(t), \quad \forall x(t) \in C .
$$

It is obvious that $G_{1}, G_{2}, b_{1}, b_{2}$ satisfy Assumption 1 and $G_{2}$ is upper semicontinuous in the first argument by the definition of the inner product $\langle.,$.$\rangle . On the other hand, we$ consider

$$
\begin{aligned}
0 & \leq G_{1}(x, y)+b_{1}(y, x)-b_{1}(x, x)+\frac{1}{r}\langle y-x, x-z\rangle \\
& =\langle y-x, x\rangle+\frac{1}{r}\langle y-x, x-z\rangle \\
& =\langle y-x,(1+r) x-z\rangle .
\end{aligned}
$$

This implies that $T_{r}^{\left(G_{1}, b_{1}\right)}(z(t))=P_{C}(z(t) /(1+r))$, $\forall z(t) \in L_{2}[0,1]$ where

$$
P_{C}(x(t))= \begin{cases}\frac{1-\langle t, x(t)\rangle}{\|t\|^{2}} t+x(t), & \text { if }\langle t, x(t)\rangle>1 \\ x(t), & \text { if }\langle t, x(t)\rangle \leq 1\end{cases}
$$

Similarly, we have $T_{r}^{\left(G_{2}, b_{2}\right)}(z(t))=P_{C}(z(t) /(1+r))$, $\forall z(t) \in L_{2}[0,1]$. For the experiments in this section, we use the Cauchy error $\left\|x_{n+1}-x_{n}\right\|^{2}<10^{-5}$ for the stopping criterion. We split considering all of the performances of our algorithm in five cases.

Case I: we start computation by comparing of the algorithm with different parameters $\bar{\theta}_{n}$ where

$$
\theta_{n}= \begin{cases}\bar{\theta}_{n}, & \text { if } n \leq N, x_{n} \neq x_{n-1}, \\ \frac{\eta_{n}}{n\left\|x_{n}-x_{n-1}\right\|}, & \text { if } n>N, x_{n} \neq x_{n-1}, \\ \eta_{n}, & \text { otherwise, }\end{cases}
$$

where $N$ is the number of iteration that we want to stop. We choose $r_{n}=0.1, \lambda_{n}=\delta_{n}=0.1, \eta_{n}=(1 /(n+1))$, and $\alpha=0.1$ and initializations $x_{0}=\sin (t)$ and $x_{1}=(\sin (t) / 2)$. Then, the results are presented as follows: Case II: we compare the performance of the algorithm with different parameters $r_{n}$ by setting $\bar{\theta}_{n}=0.5$, $\lambda_{n}=\delta_{n}=0.1, \eta_{n}=(1 /(n+1))$, and $\alpha=0.1$ and initializations $x_{0}=\sin (t)$ and $x_{1}=(\sin (t) / 2)$. Then, the results are presented as follows:

Case III: we compare the performance of the algorithm with different parameters $\eta_{n}$ by setting $\bar{\theta}_{n}=0.5, r_{n}=1$, 
TABLE 4: Numerical results of $\lambda_{n}$ and $\mu_{n}$.

\begin{tabular}{lccccc}
\hline$\lambda_{n}=\delta_{n}$ & 1 & 0.1 & 0.01 & 0.001 & 0.0001 \\
\hline No. of iters & 3 & 3 & 3 & 3 & 3 \\
CPU time (s) & 1.667450 & 1.672732 & 1.698085 & 1.663979 & 1.678559 \\
\hline
\end{tabular}

TABle 5: Numerical results of $\alpha$.

\begin{tabular}{lccccc}
\hline$\alpha$ & 0.001 & 0.1 & 0.5 & 0.9 & 7 \\
\hline No. of iters & 7 & 3 & 8 & 0.999 \\
CPU time (s) & 2.512896 & 1.663979 & 2.691693 & 2.505802 & 7 \\
\hline
\end{tabular}

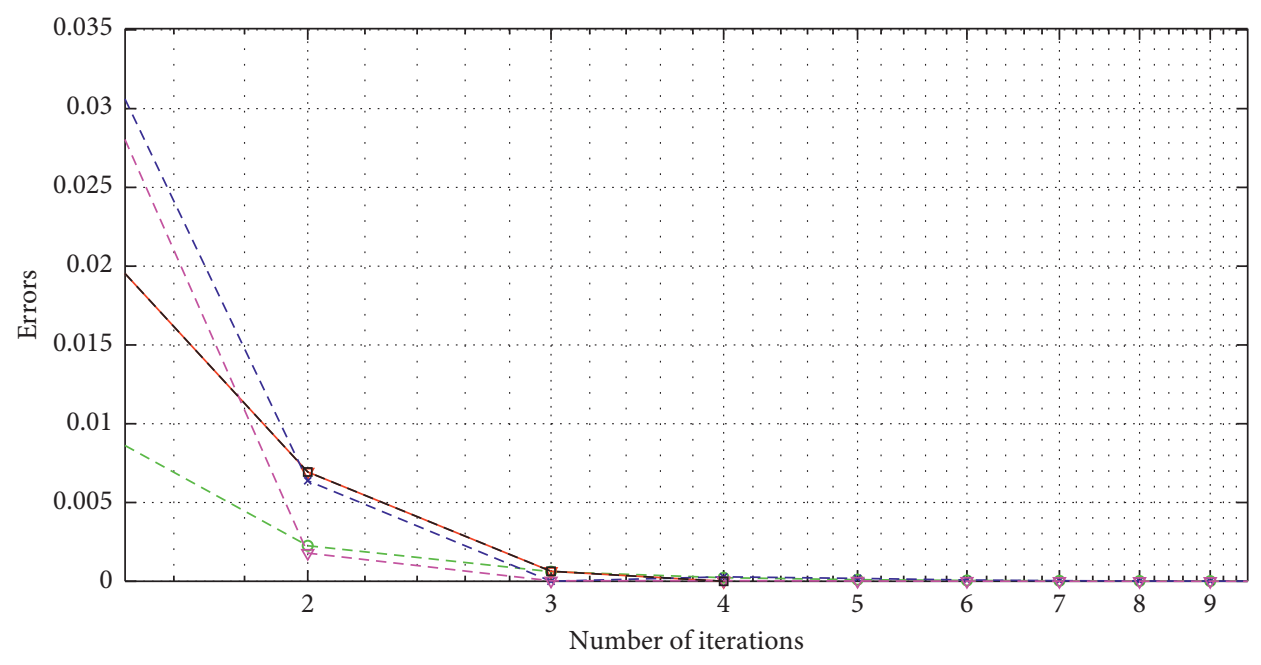

$$
\begin{array}{ll}
-\ominus-\theta_{\mathrm{n}}=0 & -\nabla-\theta_{\mathrm{n}}=1 / \mathrm{n}^{2} \\
-\nabla \theta_{\mathrm{n}}=0.5 & -\nabla-\theta_{\mathrm{n}}=\min \left\{1 / \mathrm{n}^{2}\left\|\mathrm{x}_{\mathrm{n}}-\mathrm{x}_{\mathrm{n}-1}\right\|, 0.5\right\} \\
-*-\theta_{\mathrm{n}}=1 / \mathrm{n} &
\end{array}
$$

FIgURE 1: The Cauchy error plotting number of iterations for different parameters $\bar{\theta}_{n}$.

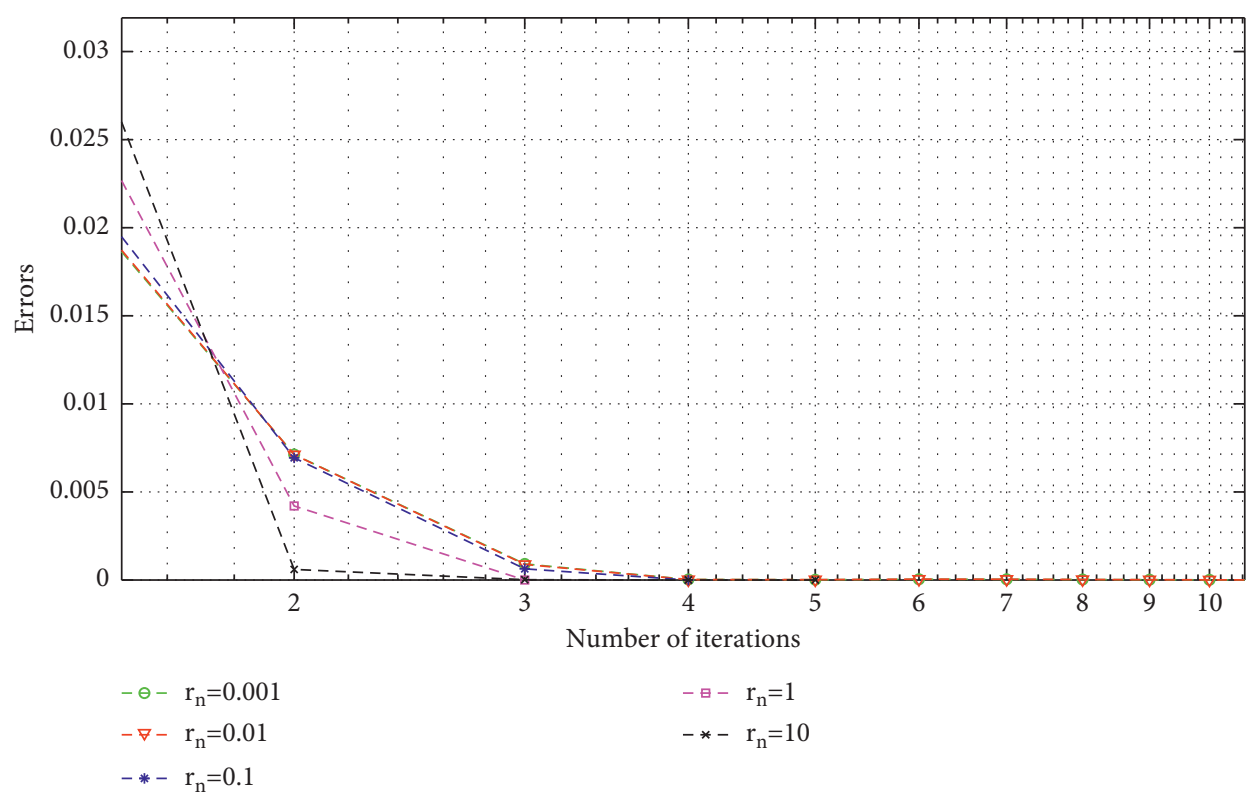

FIgURE 2: The Cauchy error plotting number of iterations for different parameters $r_{n}$. 


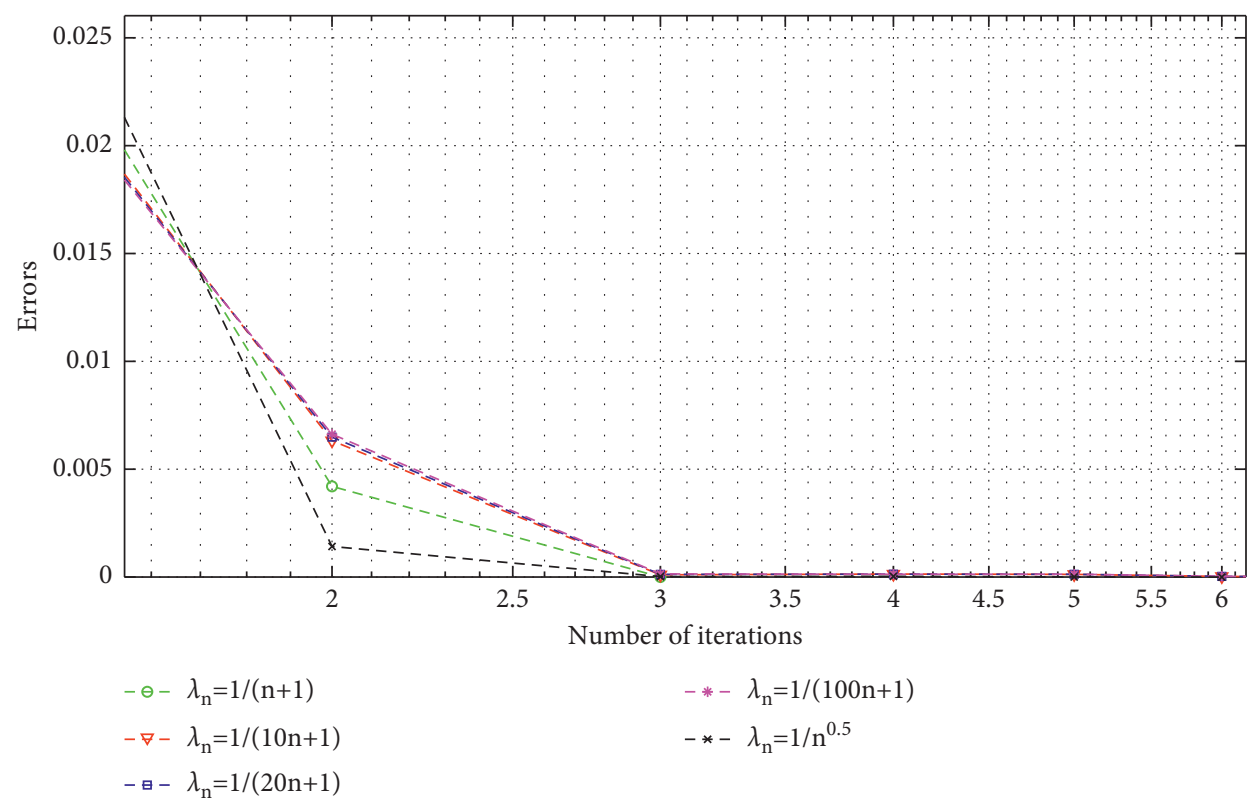

FIgURE 3: The Cauchy error plotting number of iterations for different parameters $\eta_{n}$.

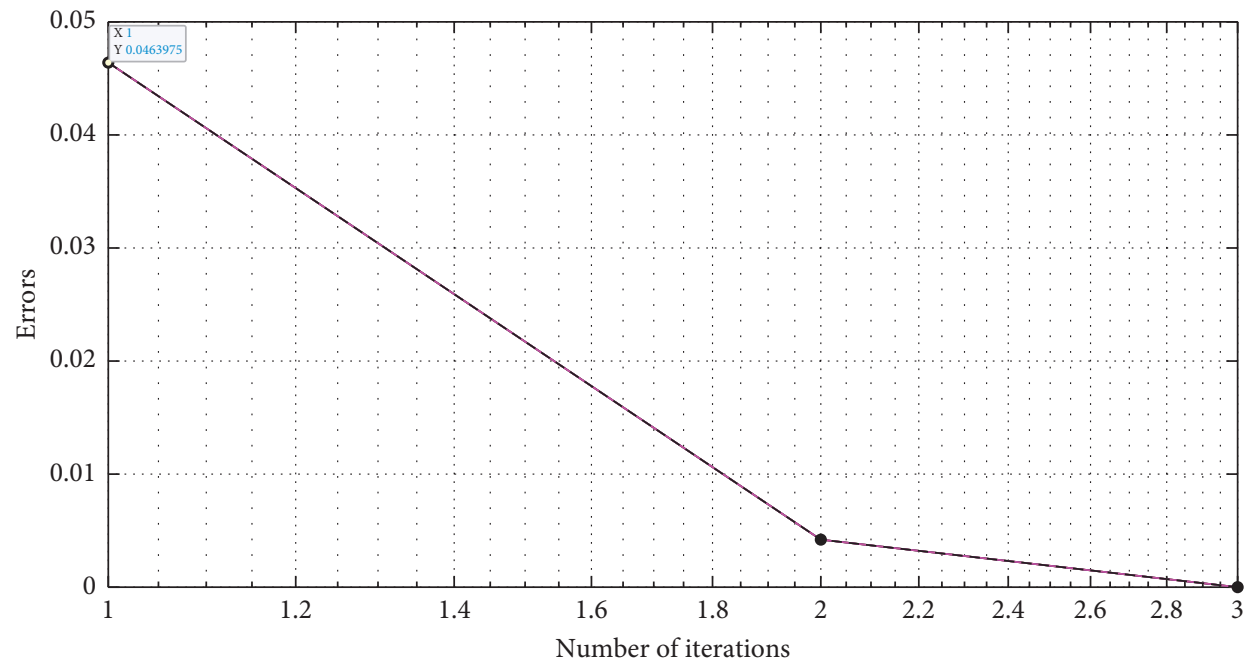

Figure 4: The Cauchy error plotting number of iterations for different parameters $\lambda_{n}$ and $\delta_{n}$.

$\lambda_{n}=\delta_{n}=0.1$, and $\alpha=0.1$ and initializations $x_{0}=\sin (t)$ and $x_{1}=(\sin (t) / 2)$. Then, the results are presented as follows:

Case IV: we compare the performance of the algorithm with different parameters $\lambda_{n}$ and $\delta_{n}$ by setting $\bar{\theta}_{n}=0.5$, $r_{n}=1, \eta_{n}=(1 /(n+1))$, and $\alpha=0.1$ and initializations $x_{0}=\sin (t)$ and $x_{1}=(\sin (t) / 2)$. Then, the results are presented as follows:
Case V: we compare the performance of the algorithm with different parameters $\alpha$ by setting $\bar{\theta}_{n}=0.5, r_{n}=1$, $\eta_{n}=(1 /(n+1))$, and $\lambda_{n}=\delta_{n}=0.001$ and initializations $x_{0}=\sin (t)$ and $x_{1}=(\sin (t) / 2)$. Then, the results are presented as follows:

From Tables $1-5$ and Figures $1-5$, we noticed that in all the above 5 cases, selecting $\vec{\theta}_{n}=0.5, r_{n}=1, \eta_{n}=(1 /(n+1))$, $\lambda_{n}=\delta_{n}=0.001$, and $\alpha=0.1$ yields the best results. 


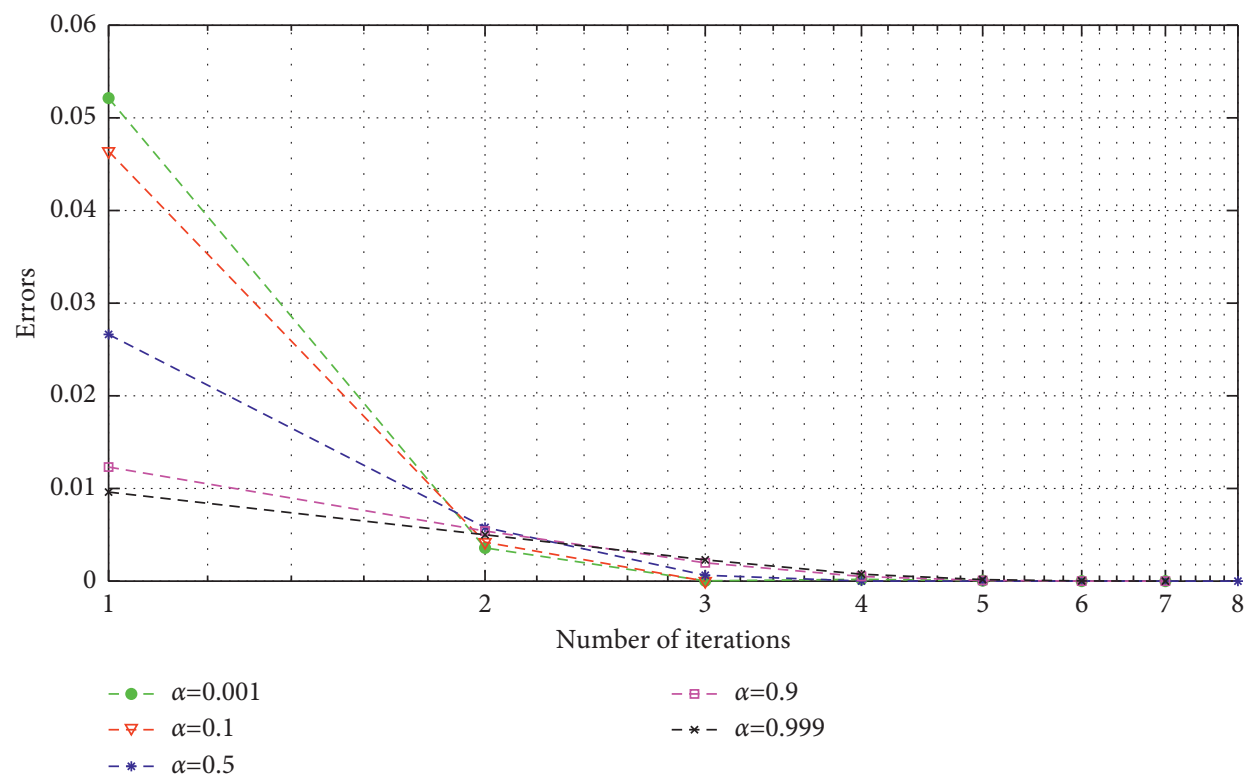

Figure 5: The Cauchy error plotting number of iterations for different parameters $\alpha$.

\section{Conclusion}

In this paper, we developed an iterative algorithm via inertial and viscosity techniques to find a common solution of a split generalized equilibrium and a variational inequality problem in Hilbert spaces. Further, we study the convergence analysis of our main result and point out some consequences. Finally, we constructed a numerical example to demonstrate the applicability of theorem and compared the performance of algorithm by taking different parameters.

\section{Data Availability}

The data used to support the findings of this study are included within the article.

\section{Conflicts of Interest}

The authors declare that they have no conflicts of interest.

\section{Authors' Contributions}

All authors contributed equally and significantly in writing this paper. All authors read and approved the final manuscript.

\section{Acknowledgments}

The researchers would like to thank the Deanship of Scientific Research, Qassim University, Kingdom of Saudi Arabia, for funding the publication of this project.

\section{References}

[1] P. Hartman and G. Stampacchia, "On some non-linear elliptic differential-functional equations," Acta Mathematica, vol. 115, pp. 271-310, 1966.
[2] E. Blum and W. Oettli, "From optimization and variational inequalities to equilibrium problems," Mathematics Student, vol. 63, pp. 123-145, 1994.

[3] L.-C. Ceng and J.-C. Yao, "A hybrid iterative scheme for mixed equilibrium problems and fixed point problems," Journal of Computational and Applied Mathematics, vol. 214, no. 1, pp. 186-201, 2008.

[4] P. L. Combettes and S. A. Hirstoaga, "Equilibrium programming in Hilbert spaces," Journal of Nonlinear and Convex Analysis, vol. 6, pp. 117-136, 2005.

[5] M. Farid, "The subgradient extragradient method for solving mixed equilibrium problems and fixed point problems in Hilbert spaces," Journal of Applied and Numerical Optimization, vol. 1, pp. 335-345, 2019.

[6] K. R. Kazmi and S. H. Rizvi, "Iterative approximation of a common solution of a split equilibrium problem, a variational inequality problem and a fixed point problem," Journal of the Egyptian Mathematical Society, vol. 21, no. 1, pp. 44-51, 2013.

[7] S. Takahashi and W. Takahashi, "Viscosity approximation methods for equilibrium problems and fixed point problems in Hilbert spaces," Journal of Mathematical Analysis and Applications, vol. 331, no. 1, pp. 506-515, 2007.

[8] Y. Censor and T. Elfving, "A multiprojection algorithm using Bregman projections in a product space," Numerical Algorithms, vol. 8, no. 2, pp. 221-239, 1994.

[9] C. Byrne, Y. Censor, A. Gibali, and S. Reich, "Weak and strong convergence of algorithms for the split common null point problem," Journal of Nonlinear and Convex Analysis, vol. 13, no. 4, pp. 759-775, 2012.

[10] Y. Censor, T. Bortfeld, B. Martin, and A. Trofimov, "A unified approach for inversion problems in intensity-modulated radiation therapy," Physics in Medicine and Biology, vol. 51, no. 10, pp. 2353-2365, 2006.

[11] Y. Censor, A. Gibali, and S. Reich, "Algorithms for the split variational inequality problem," Numerical Algorithms, vol. 59, no. 2, pp. 301-323, 2012.

[12] A. Moudafi, "The split common fixed point problem for demicontractive mappings," Inverse Problems, vol. 26, no. 5, p. 6, 2010. 
[13] P.-E. Maingé, "Convergence theorems for inertial KM-type algorithms," Journal of Computational and Applied Mathematics, vol. 219, no. 1, pp. 223-236, 2008.

[14] R. I. Bot, E. R. Csetnek, and C. Hendrich, "Inertial DouglasRachford splitting for monotone inclusion problems," Applied Mathematics and Computation, vol. 256, pp. 472-487, 2015.

[15] Q.-L. Dong, K. R. Kazmi, R. Ali, and X.-H. Li, "Inertial Krasnosel'skiľ-Mann type hybrid algorithms for solving hierarchical fixed point problems," Journal of Fixed Point Theory and Applications, vol. 21, no. 2, p. 57, 2019.

[16] Q. L. Dong, H. B. Yuan, Y. J. Cho, and T. M. Rassias, "Modified inertial Mann algorithm and inertial CQ-algorithm for nonexpansive mappings," Optimization Letters, vol. 12, no. 1, pp. 87-102, 2018.

[17] M. Farid, W. Cholamjiak, R. Ali, and K. R. Kazmi, “A new shrinking projection algorithm for a generalized mixed variational-like inequality problem and asymptotically quasi\$\$phi \$ \$-nonexpansive mapping in a Banach space," Revista de la Real Academia de Ciencias Exactas, Físicas y Naturales. Serie A. Matemáticas, vol. 115, no. 3, p. 114, 2021.

[18] S. A. Khan, S. Suantai, and W. Cholamjiak, "Shrinking projection methods involving inertial forward-backward splitting methods for inclusion problems," Revista de la Real Academia de Ciencias Exactas, Físicas y Naturales. Serie A. Matemáticas, vol. 113, no. 2, pp. 645-656, 2019.

[19] M. Alansari, R. Ali, and M. Farid, "Strong convergence of an inertial iterative algorithm for variational inequality problem, generalized equilibrium problem, and fixed point problem in a Banach space," Journal of Inequalities and Applications, vol. 2020, no. 1, p. 42, 2020.

[20] F. Alvarez, "Weak convergence of a relaxed and inertial hybrid projection-proximal point algorithm for maximal monotone operators in Hilbert space," SIAM Journal on Optimization, vol. 14, no. 3, pp. 773-782, 2004.

[21] L. Liu, S. Y. Cho, and J. C. Yao, "Convergence analysis of an inertial Tseng's extragradient algorithm for solving pseudomonotone variational inequalities and applications," Journal of Nonlinear and Variational Analysis, vol. 5, pp. 627-644, 2021.

[22] F. U. Ogbuisi, O. S. Iyiola, J. M. T. Ngnotchouye, and T. M. M. Shumba, "On inertial type self-adaptive iterative algorithms for solving pseudomonotone equilibrium problems and fixed point problems," Journal of Nonlinear Functional Analysis, vol. 20214 pages, 2021.

[23] B. T. Polyak, "Some methods of speeding up the convergence of iteration methods," USSR Computational Mathematics and Mathematical Physics, vol. 4, no. 5, pp. 1-17, 1964.

[24] A. Moudafi, "Viscosity approximation methods for fixedpoints problems," Journal of Mathematical Analysis and Applications, vol. 241, no. 1, pp. 46-55, 2000.

[25] H. H. Bauschke and P. L. Combettes, Convex Analysis and Monotone Operator Theory in Hilbert Spaces, Springer, New York, London, 2011.

[26] T. Suzuki, "Strong convergence of Krasnoselskii and Mann's type sequences for one-parameter nonexpansive semigroups without Bochner integrals," Journal of Mathematical Analysis and Applications, vol. 305, no. 1, pp. 227-239, 2005.

[27] P.-E. Maingé, "A hybrid extragradient-viscosity method for monotone operators and fixed point problems," SIAM Journal on Control and Optimization, vol. 47, no. 3, pp. 1499-1515, 2008.

[28] G. Marino and H.-K. Xu, "A general iterative method for nonexpansive mappings in Hilbert spaces," Journal of
Mathematical Analysis and Applications, vol. 318, no. 1, pp. 43-52, 2006.

[29] H.-K. Xu, "Iterative algorithms for nonlinear operators," Journal of the London Mathematical Society, vol. 66, no. 1, pp. 240-256, 2002.

[30] B. Djafari-Rouhani, K. R. Kazmi, and M. Farid, "Common solutions to some systems of variational inequalities and fixed point problems," Fixed Point Theory, vol. 18, no. 1, pp. 167-190, 2017. 